\title{
Nosocomial Infection in A Single Center of Department of Interventional Vascular Surgery- A Retrospective Analysis of 10 Years
}

\author{
Yongwei Li*1, Xuanying Qiu ${ }^{1}$, Hongjie Chen ${ }^{1}$, Zaibo Jiang ${ }^{2}$ and Hongzhi Yang ${ }^{1}$ \\ ${ }^{1}$ Department of Traditional Chinese Medicine, China \\ ${ }^{2}$ Department of Interventional Vascular Surgery, China
}

*Corresponding author: Yongwei Li, Department of Traditional Chinese Medicine, 600 Tianhe Road, Tianhe District, Guangzhou, China

\begin{tabular}{l} 
ARTICLE INFO \\
\hline Received: 幽 April 24, 2019 \\
Published: May 02, 2019 \\
Citation: Yongwei Li, Xuanying Qiu, \\
Hongjie Chen, Zaibo Jiang, Hongzhi \\
Yang. Nosocomial Infection in A Single \\
Center of Department of Interventional \\
Vascular Surgery- A Retrospective Anal- \\
ysis Of 10 Years. Biomed J Sci \& Tech Res \\
17(4)-2019. BJSTR. MS.ID.003038.
\end{tabular}

Keywords: Nosocomial Infection; Primary Liver Cancer; Transcatheter Arterial Chemoembolization

\section{ABSTRACT}

Objective: To investigate the characteristics of nosocomial infection in a department of interventional vascular surgery (DIVS) treated with transcatheter arterial chemoembolization (TACE).

Methods: The incidence of nosocomial infection was retrospectively analyzed in inpatients from January 2006 to December 2015 in DIVS.

Results: There were 10,192 inpatients in DIVS in 10 years. Nosocomial infections occurred in 341 cases. The percent of patients with nosocomial infection of the total inpatients was 3.1\%. The incidence of nosocomial infection was the lowest in PLC patients treated with TACE than the patients diagnosed liver metastasis and other vascular and biliary system-related diseases $(166 / 7862$ vs 83 / 1021 vs $72 / 1000$ patients $(p<0.01)$ ). The top three sites with the high infection rate were the peritoneum and digestive system, respiratory system, and blood system $(48.7 \%, 28.7 \%$, and $14.1 \%$, respectively). The median of hospital stay was 17 days for patients with nosocomial infections, the median time was 8 days from admission to occurrence of nosocomial infection, and the time of nosocomial infection occurred about 4 days post-surgery. Forty-three of 88 cases had positive pathogens.

Conclusion: In a single center of DIVS, the incidence of nosocomial infection was lowest in PLC patients treated with TACE. Most cases of nosocomial infection occurred in a short-term post-surgery, which could lead to prolong hospitalization duration. Pathogen examination was important to differentiate nosocomial infection from post embolism syndrome post-surgery.

\section{Introduction}

China had about 50\% primary liver cancer (PLC, mainly hepatocellular carcinoma (HCC)) of the world [1]. Transcatheter arterial chemoembolization (TACE) is the most common treatment for primary liver cancer (PLC) in China [2]. However, PLC is prone to nosocomial infection. Once infection occurs, it will limit the interventional treatment and affect the prognosis of patients [3]. But there are few reports about nosocomial infection in HCC treated with TACE. The present report was a retrospective analysis of nosocomial infection in hospitalized patients in Department of
Interventional Vascular Surgery of the Third Affiliated Hospital of Sun Yat-sen University from Jan 2006 to Dec 2015.

\section{Materials and Methods}

\section{Patients}

Inpatients in Department of Interventional Vascular Surgery (DIVS) were analyzed from January 2006-December 2015 in the Third Affiliated Hospital of Sun Yat-sen University. The diagnosis of primary disease meets diagnostic criteria for various diseases. The 
diagnosis for nosocomial infections were based on the Diagnostic Criteria for Nosocomial Infections (Trial) [4]. In brief, nosocomial infection is diagnosed by positive laboratory results of pathogen and a compatible clinical syndrome occurring 72 hours or longer after admission, or less than 72 hours after admission if patients had been admitted to the hospital before and discharged within the previous one month. The outcomes of deaths attributable to nosocomial infection were judged by a physician. TACE was carried out as the report [5]. Other invasive procedures were performed according to the normal operation guidelines. On the day postsurgery, patients were given glucose and saline, cephalosporin second- generation antibiotics to prevent infection, and protection treatment for liver function. This study was approved by the ethics committee of the hospital. Informed consent of patients was obtained prior to treatment.

\section{Methods}

A retrospective analysis method was used to review all medical records and diagnoses, general conditions and laboratory test results, nosocomial infection time, infected sites, pathogenic microorganisms, and prophylactic antibiotic use. Liver function was measured using an ALL-640 OLYMPUS automatic biochemical analyzer, blood routine examination was measured using a System XE-5000 automatic hematology analyzer, and serum hepatitis markers were detected by chemiluminescence. Pathogen culture was performed by the BD BACTECTM 9120/9240 automatic culture system (BD Biomedical Co., Ltd. Instrument Shanghai Co., Ltd.). Pathogens and drug susceptibility analysis were performed by Siemens Microscan Walkaway 96 automatic microbial analyzer.

\section{Statistical Analysis}

Data were analyzed using SPSS16.0 statistical software, and correlation analysis was performed using Spearman analysis. $\mathrm{P}<0.05$ was considered statistically significant.

\section{Results}

\section{Clinical Data}

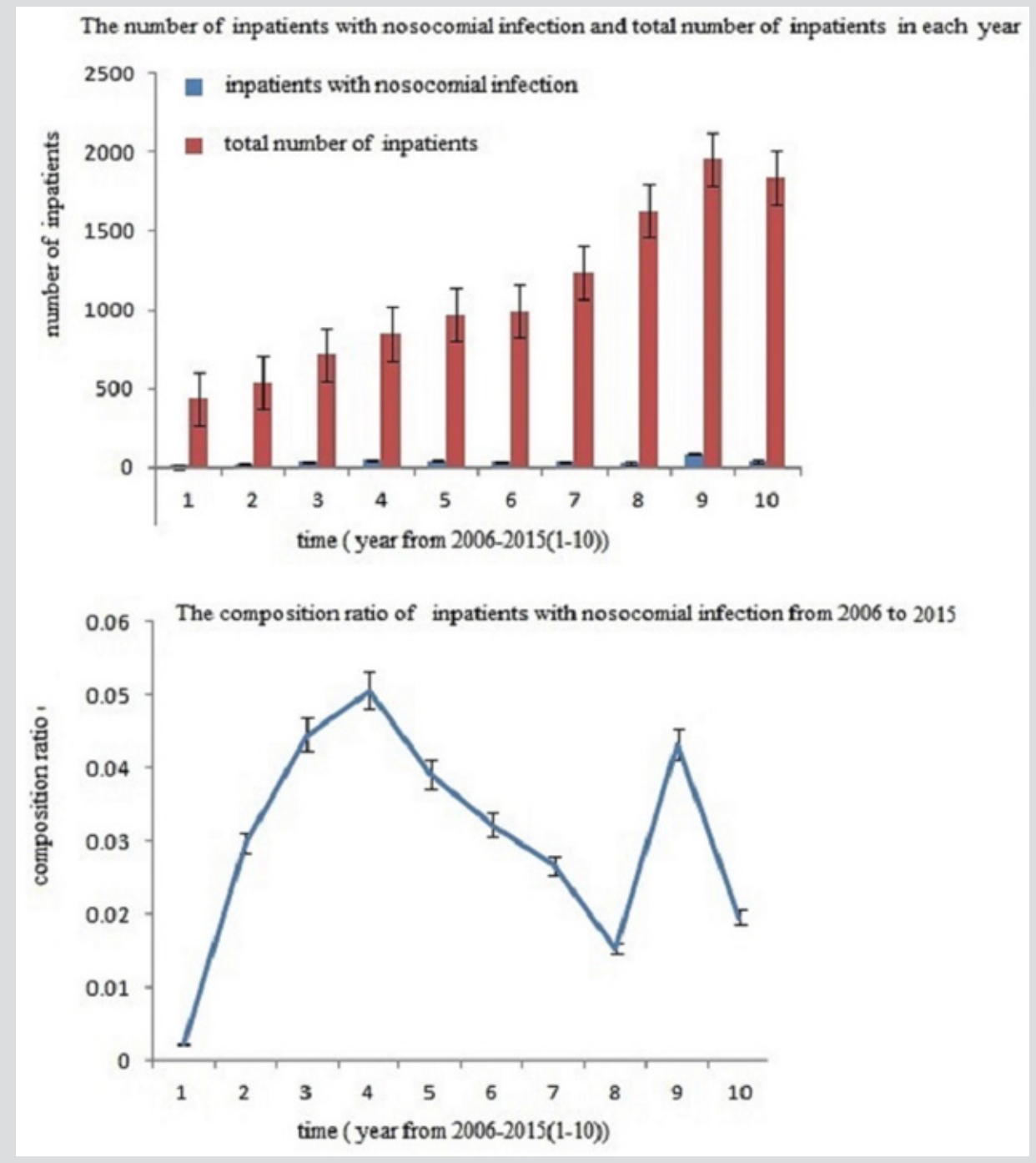

Figure 1: Distribution ratio of nosocomial infections in inpatients in Department of Interventional Vascular Surgery, Jan 2006Dec 2015. 
In the department from Jan 2006 to Dec 2015, a total of 10192 patients were hospitalized, including 7862 PLC, 1000 liver metastasis from other primary malignant lesions, and 1021 other vascular and biliary system-related diseases. The distribution ratio of nosocomial infections in inpatients in each year was shown in Figure 1. The total number of inpatients was increased from 2006 to 2015 , to which the composition ratio of nosocomial infection was not correlated ( $p>0.05$, Spearman test). The constitution ratio of nosocomial infection was $8.13 \%, 7.2 \%$, and $2.11 \%$ in other vascular and biliary system-related diseases, liver metastasis from other primary malignant lesions, and PLC, respectively (83 /1021, $72 / 1000,166 / 7862$ patients, $\mathrm{p}<0.01$, Chi-square test). Nosocomial infections were occurred in 341 cases. Three hundred and twenty-one patients occurred nosocomial infection; the percent of infected patients was $3.1 \%$. The death of nosocomial infection had no significant difference from that of non-infected patients $6 / 321$ vs 35/9871). The gender distribution was 247 males and 74 females, 16-89 years old, mean \pm standard deviation $53.1 \pm 14.3$. The top three sites with the high infection rate were the peritoneum and digestive system, respiratory system, and blood system, which were $48.7 \%, 28.7 \%$, and $14.1 \%$ (166, 98, and 48 patients), respectively. The other sites were urinary system, soft tissue, surgical site, cardiovascular and mouth $(17,6,2,2$, and 2 patients, respectively.), as shown in Figure 2.

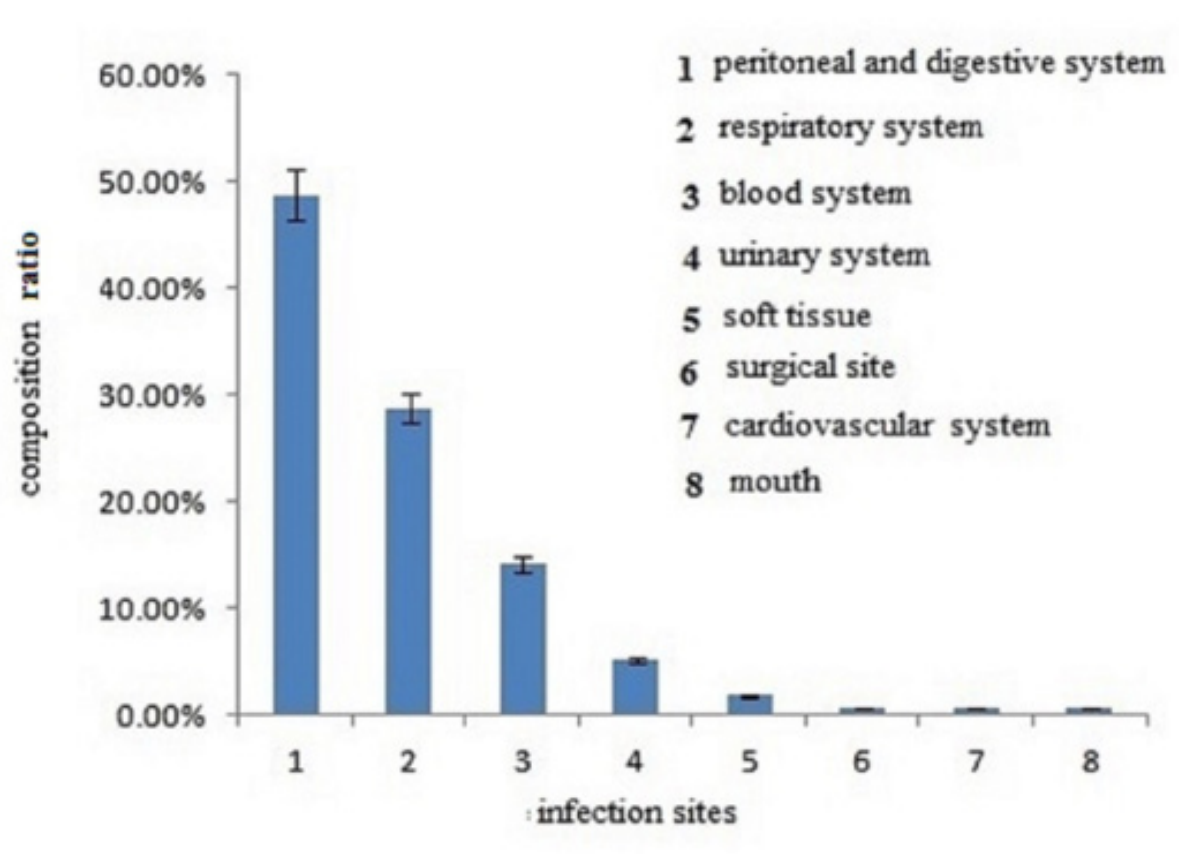

Figure 2: Composition ratio of nosocomial infection in different infection sites.

\section{Nosocomial Infection and Hospitalization Day}

The nosocomial infections occurred in 20 inpatients without any surgery or other invasive procedures. Twenty-three patients had preoperative nosocomial infections, and the remaining 278 patients had nosocomial infections post invasive procedures. The median of hospitalization duration for patients with nosocomial infections was 17 days (3-108 days), and that of total inpatients in the whole hospital was 12.8 days at the same stage (20062015). The median duration from admission to nosocomial infection occurrence was 8 days (3-102 days). Surgical patients occurred nosocomial infections about 4 days post-surgery. The total hospitalization days was positively correlated to nosocomial infection See Table 1.

Table 1: Relationship between hospitalization day and nosocomial infection duration in inpatients from Jan 2005 to Dec 2016.

\begin{tabular}{|c|c|c|c|}
\hline Name & Total hospital stays & $\begin{array}{c}\text { Duration from admission to nosocomial } \\
\text { infection }\end{array}$ & $\begin{array}{c}\text { Duration from surgery to nosocomial } \\
\text { infection }\end{array}$ \\
\hline Time (days) & $17(3-108)$ & $8(3-102)$ & $4(0-100)$ \\
\hline Correlation coefficient & & $0.733^{1}$ & $0.516^{2}$ \\
\hline P value & & 0.000 & 0.000 \\
\hline
\end{tabular}

The total number of patients was 321 with nosocomial infection, and 301 patients occurred nosocomial infection before or after invasive procedures. a) The correlation between total hospital stay and duration from admission to nosocomial infection by Spearman test, $\gamma=0.733, p=0.000$; 
b) The correlation between total hospital stay and duration from surgery to nosocomial infection by Spearman test, $\gamma=0.516, p=0.000$.

\section{Nosocomial Infection and Pathogen}

Eighty-eight samples had pathogens cultivated. Fortythree strains of pathogen were isolated from 88 samples. The distribution showed as Figure 2. There were Escherichia coli (13 cases), Klebsiella pneumonia (6 cases), Staphylococcus (6 cases), Acinetobacter baumannii (5 cases), Pseudomonas aeruginosa (5 cases), and Enterobacter cloacae (3 cases).

Other single strain included Candida glabrata, Enterococcus faecalis, Flavobacterium meningosepticum, Stenotrophomonas maltophilia, Acinetobacter haemolytica, and other gram-positive bacteria Figure 3.

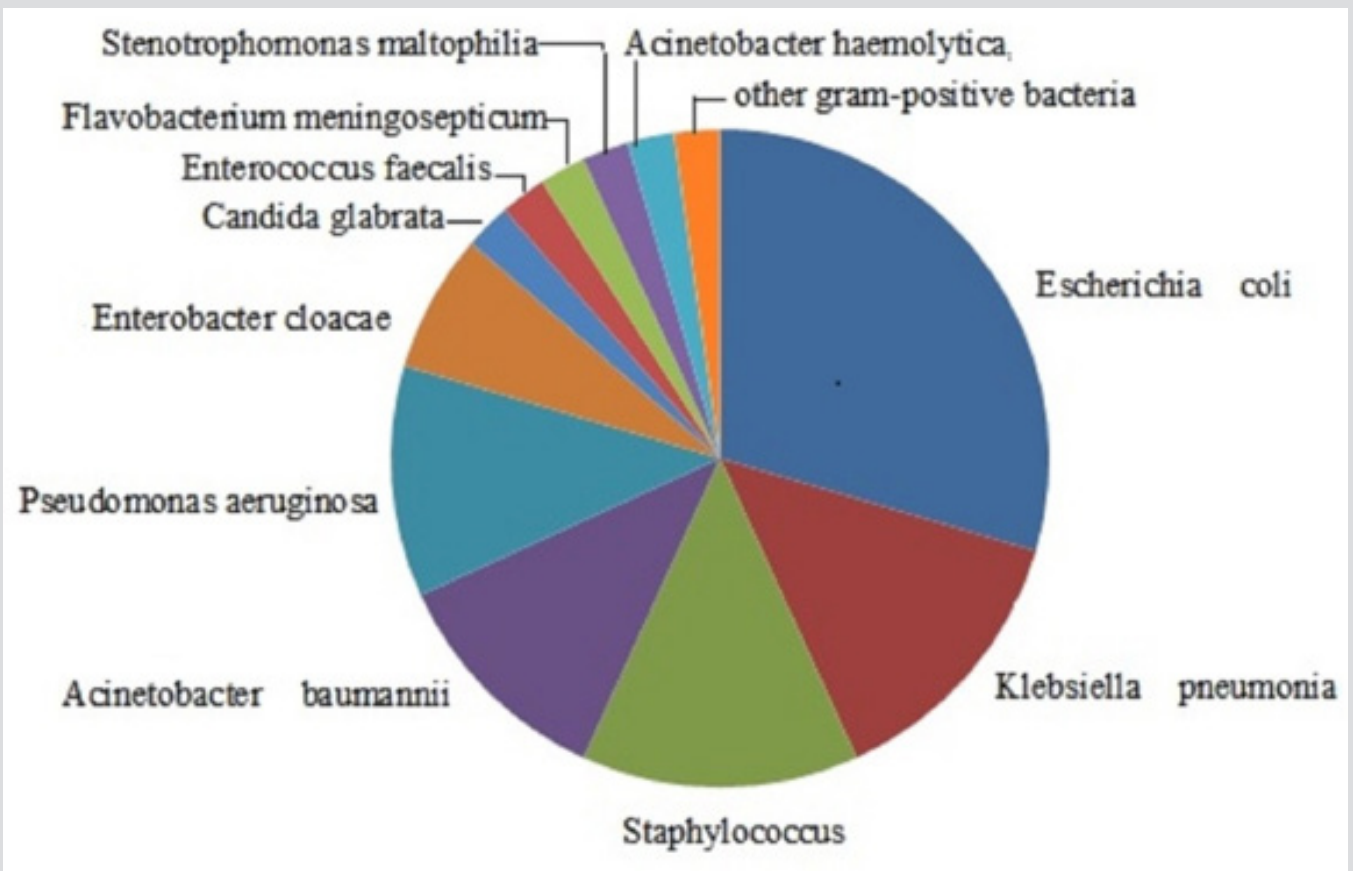

Figure 3: Pathogens in nosocomial infections.

\section{Discussion}

Interventional surgery has the advantages of fewer traumas and less complications. But PLC patients in China mostly occur based on cirrhosis. Both have high incidence of nosocomial infection. The incidence of nosocomial infection in liver cirrhosis (LC) is reported to be $22.7-47.8 \%$ [6-7], and it is 33.92\% (96/283) in late-stage PLC. The risk factors of nosocomial infection of PLC and LC are related to primary diseases, such as immune dysfunction, changes in the intestinal environment during liver cirrhosis, ascites itself an ideal medium for bacterial growth, aging, low levels of white blood cell count and albumin, poor Child-Pugh classification, etc. [6-7]. Jiang Yongjun et al. reported that the prevalence of nosocomial infection in patients undergoing primary liver cancer resection was $10.15 \%$ [8]. A small sample of liver cancer with local surgery (radiofrequency ablation) showed a nosocomial infection percent of $2.56 \%(2 / 78)$ [9]. Nosocomial infection is one of the main reasons for PLC death [3]. We searched the literatures that the prevalence of nosocomial infection of lung cancer patients was $20.1 \%$ (165/609). Age, clinical stage (IIIb and IV stage), time of hospitalization ( $\geq 22$ days), and use of hormones were predictors for nosocomial infection [10].
The nosocomial infection rate was different in PLC, liver metastasis from other primary malignant lesions, other vascular and biliary system-related diseases, maybe for patients diagnosed biliary system-related diseases such as biliary obstruction accepted operation for biliary drainage which occurred infection easily, and liver metastasis originated from other malignances had late clinical stage and more complicated symptoms than PLC. The present study showed that death between inpatients with and without infection had no significant difference. The above literatures and the results of this study (nosocomial infection rate 3.1\%) showed that nosocomial infection percentages in PLC patients undergoing surgery, especially those who had local surgery, were lower than those without surgery [3,6-9]. The reason may be related to the need for better liver function in patients receiving surgery such as TACE, that is, mostly patients at Child-Pugh A or B stage.

In this study, inpatients with nosocomial infection were mostly PLC, and the sites of nosocomial infection were mostly peritoneum and digestive system, followed by respiratory system, blood system and urinary system. Although nosocomial infection occurred mostly in inpatients post-surgery (86.6\%, 278/321patients), only 
2 cases of nosocomial infection occurred in surgical site, suggesting that the sterile operation was performed strictly, and the surgery might influence the overall immune system and induce important organs infected. The cancer lesions treated with TACE may have ischemia, necrosis, and aseptic inflammation. That might induce exudation in biliary tract and gastrointestinal tract, prone to cause dysbacteriosis of the intestinal flora and bacterial translocation, which lead to susceptibility to peritoneal and digestive infections.

Postoperative embolism syndrome occurs frequently in PLC patients treated with TACE. At least $93.4 \%$ of patients had postoperative embolism after TACE, which manifested as fever, abdomen pain, vomiting, gastrointestinal responses and other symptoms [11]. In particular, the white blood cell counts, and the percent of neutrophils may rise. And not all cultivation of pathgen are positive in nosocomial infection (43/88 samples in the present study). So, it is difficult to differentiate peritonitis or gastrointestinal tract infection from embolism syndrome. Most patients with embolism syndrome may have self-limitation, or symptomatic treatment may relieve fever, pain and vomiting [12]. While the symptom associated with hospital infection requires antibiotic treatment. However, there have no powerful differential diagnosis criteria for nosocomial infection without positive pathogens, especially for peritonitis or gastrointestinal tract infection. To improve the liver function before surgery, strictly performed aseptic procedures in invasive procedures, and administrate microecological agents, these methods need more evidence to identify the effect on nosocomial infection.

However, the duration between the admission and surgical time was generally short, and postoperative nosocomial infections occurred in a few days. Therefore, outpatients should strengthen the immunity and improve the healthy before admission. Escherichia coli were ranked at the first or second in nosocomial infections and have multi-drug resistance. The occurrence of nosocomial infection induced by production of extended-spectrum $\beta$-lactamases (ESBLs) strains has increased, of which Escherichia coli and Klebsiella pneumonia (KP) induce multidrug-resistance [13]. In our hospital, more than $50 \%$ cases of KP were resistant to ofloxacin. The data was like Taiwan that 124 (52.8\%) cases of ESBL-producing KP were resistant to ciprofloxacin [14]. The present study was showed Escherichia coli in blood and bile juice, as well as the report by Jiang Yongjun et al. [8]. The detection rates of Staphylococcus aureus, Klebsiella pneumoniae, and Acinetobacter baumannii with the increased drug resistance have also increased year by year [15].

Cephalosporins II has used usually for prevention of nosocomial infections. But above bacteria are not sensitive to the antibiotics.
Therefore, to investigate pathogens is very important prior to antibiotics administration.

In all, the treasures to reduce nosocomial infection include strictly select appropriate patients for TACE, strictly perform aseptic operations and disinfection and isolation measures, strengthen pipeline care, and monitor biochemical and blood regularly, treat underlying primary diseases.

\section{References}

1. Chen W, Zheng R, Baade PD, Zhang S, Zeng H, et al. (2016) Cancer statistics in China, 2015. CA Cancer J Clin 66(2):115- 132.

2. Diagnosis and treatment of primary liver cancer (China, 2017 edition).

3. Sun Xiaoji, Ren Liancui, Fang Xiugui (2009) Analysis and measures to prevent nosocomial infection in patients with advanced primary liver cancer. Chinese Journal of Hospital Infection 19(2): 157-159.

4. (2001) Ministry of Health of the People's Republic of China Nosocomial Infection Diagnosis criteria (Trial). Chinese Medical Journal 81(5): 314320 .

5. Miyayama S, Matsui O (2016) Superselective Conventional Transarterial Chemoembolization for Hepatocellular Carcinoma: Rationale, Technique, and Outcome. J Vasc Interv Radiol 27(9): 1269-1278.

6. Zhou Guihua, Lu Jianzhong, Li Lingen (2003) Clinical analysis of 120 cases of nosocomial infection with liver cirrhosis. Journal of Practical Liver Diseases 6(2): 96-97.

7. Guo Wanyue (2011) Clinical analysis of patients with cirrhosis complicated with nosocomial infection. Journal of Practical Liver Disease 14(6): 464-465.

8. Jiang Yongjun, Xu Xiaoxia (2011) Investigation, analysis and measures to postoperative infection in liver cancer. Pharmaceutical Forum Magazine 32(13): 95- 97.

9. Xie Xiaoxi, Lu Yinxiang, Ji Yong (2013) Causes and prevention for infection post CT-guided radiofrequency ablation in the treatment of liver cancer. Chinese Journal of Hospital Infection 23(4): 921.

10. Chen J, Pan QS, Hong WD, Pan J, Zhang WH, et al. (2014) Use of an artificial neural network to predict risk factors of nosocomial infection in lung cancer patients. Asian Pac J Cancer Prev 15(13): 5349- 5353.

11. Mondazzi L, Bottelli R, Brambilla G, Rampoldi A, Rezakovic I, et al. (1994) Transarterial Oily Chemoembolization for the treatment of Hepatocellular carcinoma: a multivariate analysis of prognostic factors. Hepatology 19(5): 1115-1123.

12. Guo Xiaohua, Zhang Wei, Jin Honglai (2010) Symptomatic treatment of interventional embolism syndrome in liver cancer (a report of 142 cases). Medical Imaging 20(7): 1000-1002.

13. Chong Y, Shimoda S, Shimono N (2018) Current epidemiology, genetic evolution and clinical impact of extended-spectrum $\beta$-lactamase- producing Escherichia coli and Klebsiella pneumoniae. Infect Genet Evol 61: 185-188.

14. Lin CJ, Siu LK, Ma L, Chang YT, Lu PL (2012) Molecular epidemiology of ciprofloxacin-resistant extended-spectrum $\beta$-lactamase- producing Klebsiella pneumoniae in Taiwan. Microb Drug Resist 18(1): 52-58.

15. Wang Shiyu, Liu Yuhua, Zhang Jianlei (2014) Analysis of the distribution and drug resistance of common pathogens in blood culture. Chinese Journal of Nosocomiology 24(1): 39-41. 


\section{ISSN: 2574-1241}

DOI: 10.26717/BJSTR.2019.17.003038

Yongwei Li. Biomed J Sci \& Tech Res

(C) (i) This work is licensed under Creative

Submission Link: https://biomedres.us/submit-manuscript.php

$\begin{array}{ll}\text { BIOMEDICAL } & \text { Assets of Publishing with us } \\ \text { RESEARCHES } & \text { - Global archiving of articles } \\ \text { - Immediate, unrestricted online access } \\ \text { - Rigorous Peer Review Process }\end{array}$

